\title{
Liposomes bearing fibrinogen could potentially interfere with platelet interaction and procoagulant activity
}

This article was published in the following Dove Press journal:

International Journal of Nanomedicine

9 May 2012

Number of times this article has been viewed

\section{Rosa Hernández \\ Patricia Urbán ${ }^{2}$ \\ Elisenda Casals ${ }^{3}$ \\ Joan Estelrich ${ }^{3}$ \\ Ginés Escolar' \\ Ana M Galán'}

'Department of Hemotherapy and Hemostasis, Hospital Clinic, CDB, IDIBAPS, UB, Barcelona, Spain; ${ }^{2}$ Nanobioengineering Group, Institute for Bioengineering of Catalonia, Barcelona, Spain; ${ }^{3}$ Department of Physical Chemistry, Faculty of Pharmacy, University of Barcelona, Barcelona, Spain
Correspondence: Ana M Galán Servicio de Hemoterapia y Hemostasia, Hospital Clínic, IDIBAPS, Villarroel,

170 08036-Barcelona, Spain

Tel +34 932275400 ext 2034; 2307; 2306

Fax +34932279369

Email agalan@clinic.ub.es
Background: The contribution of fibrinogen (FBN) to hemostasis acting on platelet aggregation and clot formation is well established. It has been suggested that FBN-coated liposomes could be useful in restoring hemostasis. In the present study, we evaluated the modifications induced by multilamellar raw liposomes (MLV) or fibrinogen-coated liposomes (MLV-FBN) on hemostatic parameters.

Materials and methods: Different experimental settings using whole blood or thrombocytopenic blood were used. Thromboelastometry, aggregation studies, platelet function analyzer $\left(\right.$ PFA- $\left.100^{\circledR}\right)$ tests and studies under flow conditions were applied to detect the effect of MLVFBN on hemostatic parameters.

Results: The presence of MLV-FBN in whole blood modified its viscoelastic properties, prolonging clot formation time (CFT) $(226.5 \pm 26.1 \mathrm{~mm}$ versus $124.1 \pm 9.4 \mathrm{~mm} ; P<0.01)$ but reducing clot firmness $(45.4 \pm 1.8 \mathrm{~mm}$ versus $35.5 \pm 2.3 \mathrm{~mm} ; P<0.05)$. Under thrombocytopenic conditions, FIBTEM analysis revealed that MLV-FBN shortened clotting time (CT) compared to MLV $(153.3 \pm 2.8 \mathrm{~s}$ versus $128.0 \pm 4.6 \mathrm{~s} ; P<0.05)$. Addition of either liposome decreased fibrin formation on the subendothelium (MLV 8.1\% $\pm 4.7 \%$ and MLV-FBN $0.8 \% \pm 0.5 \%$ versus control $36.4 \% \pm 6.7 \% ; P<0.01$ ), whereas only MLV-FBN significantly reduced fibrin deposition in thrombocytopenic blood $(14.4 \% \pm 6.3 \%$ versus control $34.5 \% \pm 5.2 \% ; P<0.05)$. MLV-FBN inhibited aggregation induced by arachidonic acid $(52.1 \% \pm 8.1 \%$ versus $88.0 \% \pm 2.1 \%$ in control; $P<0.01)$ and ristocetin $(40.3 \% \pm 8.8 \%$ versus $94.3 \% \pm 1.1 \% ; P<0.005)$, but it did not modify closure times in PFA- $100^{\circledR}$ studies. In perfusion experiments using whole blood, MLV and MLV-FBN decreased the covered surface (13.25\% $\pm 2.4 \%$ and $9.85 \% \pm 2.41 \%$, respectively, versus control $22.0 \% \pm 2.0 \% ; P<0.01)$ and the percentage of large aggregates $(8.4 \% \pm 2.3 \%$ and $3.3 \% \pm 1.01 \%$, respectively, versus control $14.6 \% \pm 1.8 \% ; P<0.01$ ).

Conclusion: Our results reveal that, in addition to the main contribution of fibrinogen to hemostasis, MLV-FBN inhibits platelet-mediated hemostasis and coagulation mechanisms.

Keywords: thrombocytopenia, hemostasis, fibrinogen, liposomes, procoagulant activity, fibrin

\section{Introduction}

Liposomes are widely implemented for therapeutic use as drug carriers, as well as in drug targeting and other pharmacological applications, including basic research and applied technology. ${ }^{1,2}$ In previous studies, our group demonstrated the development of procoagulant activity in damaged vessels of synthetic phospholipids and liposomes, ${ }^{3,4}$ opening up new possibilities for using liposomes as platelet substitutes to improve hemostasis. Several groups have explored the use of modified liposomes and microcapsules as coagulants in order to improve hemostasis. ${ }^{3-9}$ 
Recently, the use of nanotechnological approaches to stop bleeding has been explored. ${ }^{10}$ In this context, the effects of prohemostatic molecules, such as clotting factors in liposomes, could provide many advantages in controlling hemorrhages.

Fibrinogen $(\mathrm{FBN})$ is a $340 \mathrm{kDa}$ protein that is present in plasma at a concentration of 2 to $3 \mathrm{mg} / \mathrm{mL}$. It has two identical disulfide-linked subunits composed of three nonidentical polypeptide chains: $A \alpha, B \beta$, and $\gamma$. In addition, a $\gamma^{\prime}$ chain is found in approximately $10 \%$ of FBN molecules. The four carboxyl-terminal amino acids found in the $\gamma \mathrm{A}$ chain are substituted by a highly anionic 20-amino acid sequence in the $\gamma^{\prime}$ chain. ${ }^{11}$ FBN plays an important role in blood coagulation as the substrate for thrombin, which is the final step in the coagulation cascade that results in fibrin formation. ${ }^{12}$ Moreover, FBN mediates platelet aggregation by binding and bridging glycoprotein IIb-IIIa (GPIIb-IIIa). ${ }^{13}$ Two regions of the FBN $\alpha$ chain that contain an arginine-glycine-aspartic acid motif, as well as the carboxyl-terminus of the FBN $\gamma$ chain, represent potential binding sites for GPIIb-IIIa in the fibrinogen molecule. ${ }^{14,15}$

Thus, combining adequate sequences of adhesive proteins in the correct conformation on the appropriate carrier could improve primary hemostasis without enhancing fibrin formation. In the present study, we explored the effects of both raw multilamellar liposomes (MLV) and fibrinogencoated liposomes (MLV-FBN) on platelet function by using different experimental approaches: platelet aggregation induced by different agonists; experiments under flow conditions using the platelet function analyzer PFA- $100^{\circledR}$ (Siemens Healthcare Diagnostics, Deerfield, IL) device; and perfusion systems to evaluate platelet interaction and fibrin formation on the subendothelium. Moreover, viscoelastic properties and the quality of clot formation were tested by thromboelastometric techniques.

\section{Materials and methods Experimental design}

The present study was designed to evaluate the possible effect of liposomes containing FBN on different hemostatic parameters. The activity of liposomes was evaluated using whole blood and mild thrombocytopenic blood $(30,000$ platelets/ $\mu \mathrm{L})$ anticoagulated with low-molecular-weight heparin (LMWH) or citrate $(19 \mathrm{mM})$, depending on the test used. Blood samples were incubated with MLV or MLV-FBN liposomes and the effects on the coagulation system, platelet activation, thrombus formation, and fibrin generation were assessed by different experimental settings.

\section{Reagents}

1,2-dipalmitoyl-sn-glycero-3-phosphoethanolamine (PE) and 1,2-dipalmitoyl-sn-glycero-3-phosphoethanolamine$\mathrm{N}$-[4-(p-maleimidophenyl)butyramide] (N-MPB-PE) were purchased from Avanti Polar Lipids (Alabaster, AL); 1,2-dipalmitoyl-rac-glycero-3-phosphocholine (DPPC), cholesterol (CHOL), and FBN from human plasma protein from Sigma-Aldrich (St Louis, MO); and $\mathrm{N}$-succinimidyl-S-acetylthioacetate (SATA) from Pierce (Rockford,IL). Thebufferusedwas50mM4-(2-hydroxyethyl)-1piperazine ethanesulphonic acid (HEPES), pH 7.5 (with and without $1 \mathrm{mM}$ ethylenediaminetetraacetic acid for liposomes with and without FBN). Other reagents used were of analytical grade. The water used was ultrapure (MilliQ reverse osmosis system; Millipore, Billerica, MA) with a resistivity of $18.3 \mathrm{M} \Omega / \mathrm{cm}$. Arachidonic acid and ristocetin were purchased from Helena BioSciences Europe (Gateshead, UK), while collagen and adenosine diphosphate (ADP) were from Arkray Inc (Kyoto, Japan). ROTEM ${ }^{\circledR}$ reagents for the EXTEM and FIBTEM tests were from Pentapharm $\mathrm{GmbH}$ (Aesch, Switzerland).

\section{Liposome preparation and characterization}

Phospholipids (DPPC:CHOL:N-MPB-PE and DPPC:CHOL:PE at a molar ratio of 50:20:30) were dissolved in chloroform in a round-bottomed flask and dried in a rotary evaporator under reduced pressure at $50^{\circ} \mathrm{C}$ to form a thin film on the flask. The film was hydrated with a solution of $10 \mathrm{mM}$ HEPES buffer ( $\mathrm{pH} 7.4)$ and $145 \mathrm{mM}$ $\mathrm{NaCl}$ to give a lipid concentration of $20 \mathrm{mM}$. Multilamellar liposomes were formed by constant vortexing for 4 minutes on a vortex mixer followed by sonication for 4 minutes in a Transsonic Digital T460/H bath sonicator (Elma ${ }^{\circledR}$, Singen, Germany) to guarantee the complete suspension of lipids. Vesicle size, size distribution and $\zeta$-potential were determined at $25^{\circ} \mathrm{C}$ by photon correlation spectroscopy and Doppler microelectrophoresis, using a Zetasizer Nano ZS90 (Malvern Instruments, Malvern, UK). ${ }^{16}$

\section{Preparation of thiolated fibrinogen and coupling to liposomes}

The procedure for thiolating proteins with SATA has been previously reported. ${ }^{17}$ The number of introduced thiol groups was assayed according to Ellman, using cysteine for the calibration curve. ${ }^{18}$ According to this assay, the number of introduced thiol groups (expressed as mol of SH/mol of $\mathrm{FBN}$ ) was $1.6 \pm 0.1$. 
A volume of liposome suspension (DPPC: N-MPB-PE) was combined with an equal volume of fibrinogen solution. The resulting suspension was left to react overnight at room temperature while being centrifuged at 1,000 rpm.

The coupling reaction was stopped by adding $50 \mu \mathrm{L}$ of N-ethylmaleimide ( $8 \mathrm{mM}$ in HEPES buffer) and the liposomes were separated from free protein by two centrifugation steps in Optima L-100XP (Beckman-Coulter, Fullerton, CA) for 40 minutes at $100,000 \mathrm{~g}$ and $7^{\circ} \mathrm{C}$. Liposomes (with or without FBM) were prepared in different batches. Measurements of size, $\zeta$-potential, and amount of thiol groups introduced on the liposomal surface served as control parameters to ensure that the physicochemical properties were the same in all batches used.

\section{Blood collection and preparation of thrombocytopenic blood}

Blood was obtained from six healthy volunteers (4 males and 2 females, with an age range from 20 to 65 years) who had not taken drugs affecting platelets or coagulation mechanisms in the previous 10 days. Prior to the study, our local institutional ethics committee approved the protocol, which conformed to the ethical guidelines of the Helsinki Declaration. Written informed consent was obtained from all subjects. Blood was anticoagulated with LMWH (Fragmin ${ }^{\circledR}$; Pfizer Inc, New York, $\mathrm{NY}$ ) at a final concentration of $20 \mathrm{U} / \mathrm{mL}$. This concentration of LMWH allows the generation of thrombin when blood is exposed to a denuded vascular segment. ${ }^{19-22}$ Blood platelets and leukocytes were reduced by a filtration procedure, ${ }^{23}$ using an RC100 filter (PALL Corp, Glen Cove, NY). The platelet number was adjusted to 30,000 platelets $/ \mu \mathrm{L}$, mixing thrombocytopenic blood and whole blood. Before each experiment, samples were preincubated with liposomes coupled or uncoupled to $\mathrm{FBN}$ for $2 \mathrm{~min}$ at $37^{\circ} \mathrm{C}$. PFA- $100^{\circledR}$ and aggregation experiments were performed with blood anticoagulated by citrate at a final concentration of $19 \mathrm{mM}$ using standard laboratory procedures.

\section{Thromboelastometric studies}

To examine the kinetics and quality of clot formation, we used the ROTEM ${ }^{\circledR}$ thromboelastometry analyzer. ${ }^{24}$ The technique was performed according to the manufacturer's instructions. We used two different tests: EXTEM and FIBTEM. In the EXTEM test, tissue factor (TF) is used as an activator and is sensitive to changes in the extrinsic pathway of coagulation, FBN and fibrin polymerization, and platelet function. In the FIBTEM test, platelet function is inhibited by the platelet inhibitor cytochalasin D. While the clots obtained in EXTEM were formed by platelets and fibrin, the clot obtained in the FIBTEM assay was primarily a fibrin clot.

The study was carried out with whole and thrombocytopenic blood (30,000 platelets $/ \mu \mathrm{L})$ using $300 \mu \mathrm{L}$ of blood containing $10 \%$ liposomes coupled or uncoupled to FBN. Measurements were recorded for 30 minutes, while controls were performed simultaneously by adding $10 \%$ of the corresponding buffer to the blood.

The main effect of liposomes was measured during clot formation by the standard thrombelastometric parameters: clotting time (CT) is the period of time from the start of the analysis until the clot reached $2 \mathrm{~mm}$ in amplitude; clot formation time (CFT) is the period until $20 \mathrm{~mm}$ amplitude was reached; and clot firmness was measured at 10-minute intervals (A10). ${ }^{25}$ The CT and CFT, measured in seconds, indicate the dynamics of clot formation. The clot amplitude $(\mathrm{mm})$ gives information about clot strength and stability, which largely depends on FBN and platelets.

\section{Platelet aggregation}

The experiments were performed in a four-channel aggregometer (APACT 4, Helena BioSciences) according to the classical turbidimetric technique. ${ }^{26}$ Aliquots of $450 \mu \mathrm{L}$ of platelet-rich plasma were transferred to the aggregometer cuvette and activated with different agonists: arachidonic acid and ristocetin (Helena BioSciences) at a final concentration of $1.4 \mathrm{mM}$ and $1 \mathrm{mg} / \mathrm{mL}$, respectively, and collagen and ADP at a final concentration of $2.5 \mu \mathrm{g} / \mathrm{mL}$ and $2 \mu \mathrm{M}$, respectively. The values of maximal platelet aggregation were recorded and expressed as percentages.

\section{PFA- $100^{\circledR}$ studies}

PFA- $100^{\circledR}$ evaluates the hemostatic capacity of platelets as a function of their ability to occlude an aperture in a membrane coated with collagen and epinephrine (EPI) or collagen and ADP under high shear stress $\left(5000 \mathrm{~s}^{-1}\right) \cdot{ }^{27}$ Normality values for closure times previously established for the PFA- $100^{\circledR}$ in our laboratory were $113 \pm 24 \mathrm{~s}$ (mean \pm standard deviation (SD)) with cartridges containing collagen and epinephrin (COL-EPI) and $87 \pm 18 \mathrm{~s}$ with cartridges containing collagen and adenosin diphosphate (COL-ADP). ${ }^{21}$ Aliquots of blood incubated with MLV or MLV-FBN were used to study potential changes in closure time.

\section{Perfusion studies}

Rabbit abdominal aortic segments were prepared and processed as previously described. ${ }^{28}$ Everted segments were mounted on a rod and inserted into the annular chamber. 
Two-mL aliquots of each liposome preparation were added to the blood samples. Control perfusions were carried out by adding equivalent amounts of the buffer used in the phospholipid preparations. The perfusates, with a total volume of $20 \mathrm{~mL}$, were incubated at a constant temperature of $37^{\circ} \mathrm{C}$ for 2 minutes before starting the perfusion. Perfusions were conducted in annular chambers according to the method previously described. ${ }^{3,4,28}$ Blood was recirculated through the chamber for 10 minutes at $37^{\circ} \mathrm{C}$ at a shear rate of $250 \mathrm{~s}^{-1}$, using a peristaltic pump. This low shear rate facilitates fibrin deposition onto the subendothelium.

At the end of the perfusion, vessel segments were histologically processed. Platelet interaction and fibrin deposition onto perfused subendothelium were analyzed morphometrically as previously described. Platelet interaction was expressed as the percentage of covered surface by platelets and the percentage of big platelet aggregates $(>5 \mu \mathrm{m})$ classified as $\%$ thrombi $(\% \mathrm{~T})$. The presence of fibrin was expressed as a percentage of fibrin..$^{20,29,30}$

\section{Data analysis}

Results are expressed as mean \pm standard error of the mean (SEM). The number of experiments for each preparation was at least $n=6$. Student's $t$-test for paired data was used to compare differences between the control and the treated samples. The level of statistical significance was established at $P<0.05$.

\section{Results}

\section{Liposome characterization}

The average size of liposomes, irrespective of the presence of FBN, ranged from 0.8 to $1.4 \mu \mathrm{m}$, and their polydispersity was close to 0.80 . The incubation ratio of SATA to FBN of 10 (molar ratio) resulted in the introduction of 1.5 to $1.7 \mathrm{~mol}$ of thiol groups/mol of FBN. FBN was coupled at a ratio of 33 to $53 \mu \mathrm{g}$ of FBN per $\mu \mathrm{mol}$ of phospholipid in the different FBN-MLV batches tested.

Measurements of the $\zeta$-potential of raw liposomes in water afforded an average value of $-8 \mathrm{mV}$, whereas after the coupling of fibrinogen, this value increased to $-75 \mathrm{mV}$.

\section{Effect of liposome preparation on hemostatic parameters}

\section{Thromboelastometric studies}

The addition of MLV-FBN to whole blood did not modify the $\mathrm{CT}$ in comparison with the control in the EXTEM analysis. However, the presence of MLV-FBN led to a significant increase in the CFT $(226.5 \mathrm{~s} \pm 26.13$ versus $124.17 \mathrm{~s} \pm 9.46$;
$P<0.01$, indicating a certain inhibition of clot formation. Moreover, MLV-FBN reduced clot firmness, measured as A10, with respect to control $(35.50 \pm 2.38 \mathrm{~mm}$ versus $45.40 \pm 1.82 \mathrm{~mm} ; P<0.05)$. These results are summarized in Figure 1. No differences were observed when the experiments were performed using thrombocytopenic blood.

The analysis of parameters using the FIBTEM tests indicated that the addition of MLV-FBN to whole blood did not induce differences compared to the control. However, in experiments performed with thrombocytopenic blood, MLVFBN decreased the CT with respect to MLV (153.33 \pm 2.82 versus $128.00 \pm 4.62 ; P<0.05)$, whereas no differences were found between MLV-FBN and control (Figure 1).

As expected, the results using whole blood in the EXTEM test showed a reduction in clot formation and clot firmness. This test reflected the role of platelets and coagulation processes in hemostasis. In contrast, results obtained with the FIBTEM tests, which eliminated platelet function, showed significant differences in thrombocytopenic blood.

\section{Platelet aggregation studies}

MLV modified aggregation profiles only when arachidonic acid was used as an agonist $(50.9 \% \pm 7.9 \%$ versus $88.0 \% \pm 2.1 \%$ in control; $P<0.01$ ). Interestingly, MLVFBN also inhibited aggregation induced by arachidonic acid $(52.1 \% \pm 8.1 \%$ versus $88.0 \% \pm 2.1 \%$ in control; $P<0.01)$ and ristocetin $(16.7 \pm 3.2$ versus $68.3 \% \pm 5.8 \% ; P<0.01)$. Aggregations induced by ADP and collagen were not modified by either MLV or MLV-FBN. However, we observed a reduction in the maximal aggregation induced by ADP in the case of MLV-FBN, but this was not statistically significant. These results are summarized in Figure 2.

\section{PFA- $100^{\circledR}$ studies}

The addition of liposomes to whole blood did not statistically modify closure times in the PFA- $100^{\circledR}$ device. COL-EPI cartridges showed values of $171 \pm 48.1 \mathrm{~s}$ for MLV, $194 \pm 27.3 \mathrm{~s}$ for MLV-FBN, and $184 \pm 27.4 \mathrm{~s}$ for control. Results using COL-ADP cartridges were $113.5 \mathrm{~s} \pm 21.6 \mathrm{~s}$ for MLV, $124.3 \pm 20.5 \mathrm{~s}$ for MLV-FBN, and $95 \pm 16.9 \mathrm{~s}$ for control.

\section{Perfusion experiments}

Perfusions with whole blood resulted in a platelet coverage surface of $22.01 \% \pm 2.05 \%$. Addition of MLV and MLV-FBN to whole blood samples decreased the coverage surface of platelets $(13.25 \% \pm 2.4 \%$ and $9.85 \% \pm 2.41 \%$, respectively, versus $22.01 \% \pm 2.05 \% ; P<0.01)$. A more detailed analysis of platelet interaction also showed a decrease in the presence 


\section{EXTEM}

A
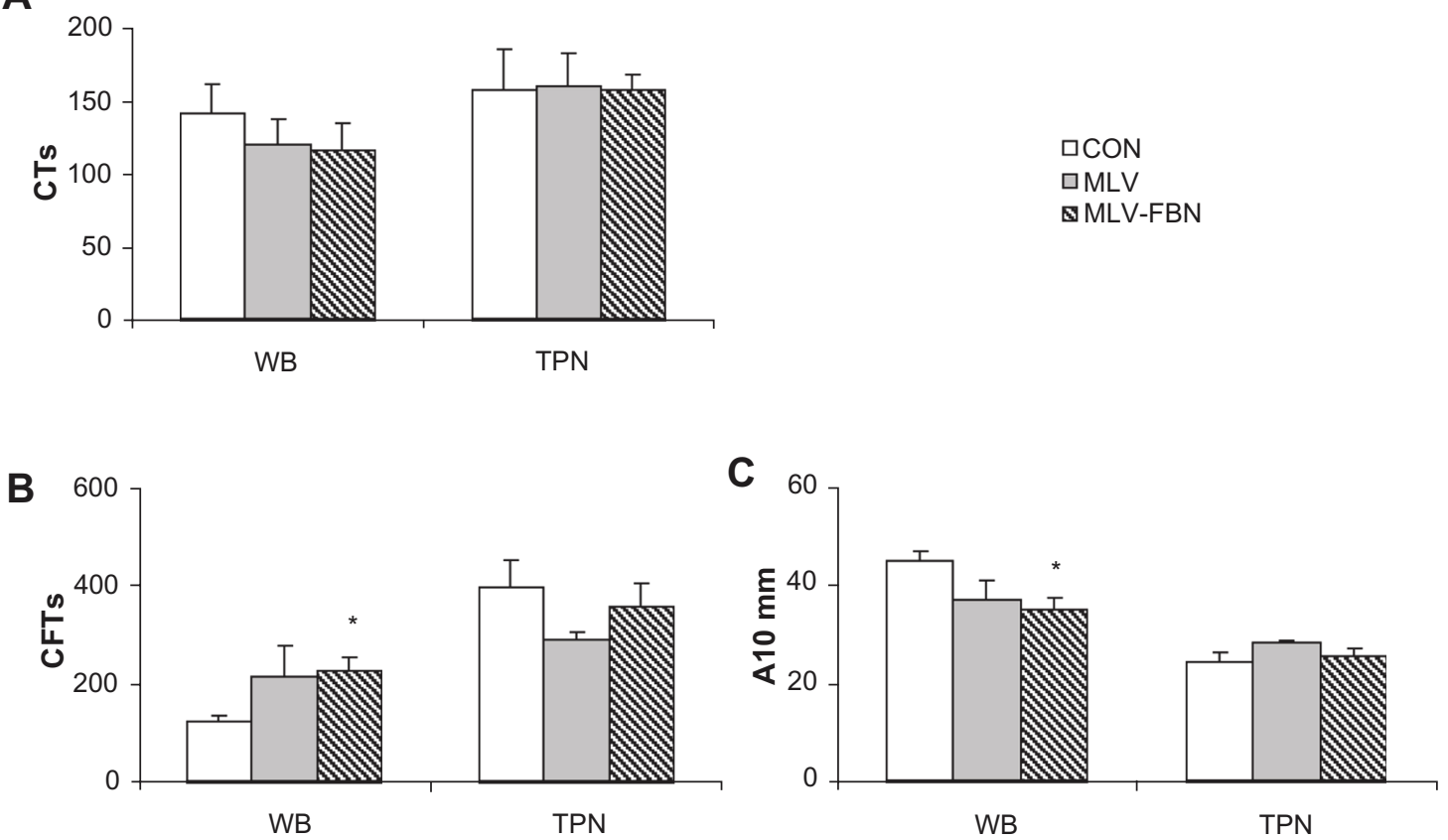

FIBTEM
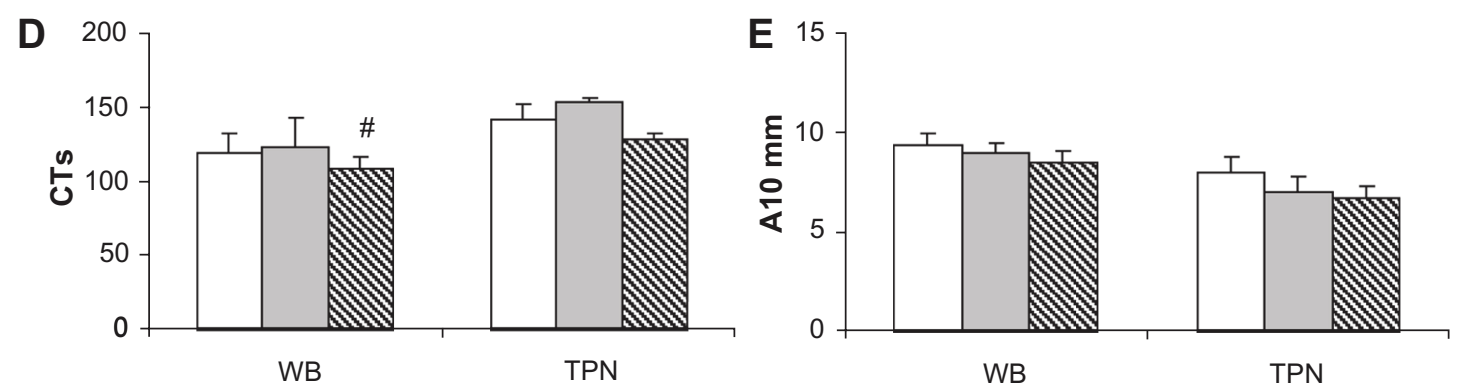

Figure I Thromboelastometric results of EXTEM- (A-C) and FIBTEM (D and E) derived parameters (CT and CFT expressed in s, and AI0 in mm). Experiments were performed in whole blood (WB) and thrombocytopenic blood (TPN) following in vitro addition of MLV and MLV-FBN.

Notes: Data presented as mean \pm SEM, $n=6$. $*$ MLV-FBN versus control $P<0.01$; ${ }^{\# M L V-F B N ~ v e r s u s ~} M L V$ liposomes $P<0.01$.

Abbreviations: CT, clotting time; CFT, clot formation time; CON, control; MLV, raw multilamellar liposomes; MLV-FBN, fibrinogen-coated liposomes; SEM, standard error of the mean.

of large aggregates measured as $\% \mathrm{~T}(8.44 \% \pm 2.33 \%$ and $3.36 \% \pm 1.01 \%$, respectively, versus $14.64 \% \pm 1.89 \%$ in control experiments; $P<0.01$ ). Moreover, a significant decrease in fibrin deposition was observed when liposomes were present in perfusates (MLV 8.12\% $\pm 4.73 \%$ and MLV-FBN $0.84 \% \pm 0.52 \%$ versus control $36.48 \% \pm 6.76 \% ; P<0.01$ ) Representative microscopic fields are shown in Figure 3.

Experiments performed under conditions of moderate thrombocytopenia revealed that the presence of liposomes resulted in a significant reduction $(P<0.05)$ of the percentage of covered surface by platelets (MLV $7.30 \% \pm 1.17 \%$ and
MLV-FBN $6.99 \% \pm 0.94 \%$ versus $12.50 \% \pm 1.31 \%$ in thrombocytopenic blood; $P<0.05$ ). MLV did not modify fibrin deposition on the subendothelium $(34.58 \% \pm 5.21 \%)$, but there was a significant decrease in fibrin deposition after the addition of MLV-FBN to thrombocytopenic blood $(14.47 \% \pm 6.34 \%$; $P<0.05)$. These results are summarized in Figure 4.

\section{Discussion}

The present study focused on the preparation of FBN-coated liposomes in order to improve hemostatic mechanisms, such as platelet reactivity and coagulation mechanisms. 


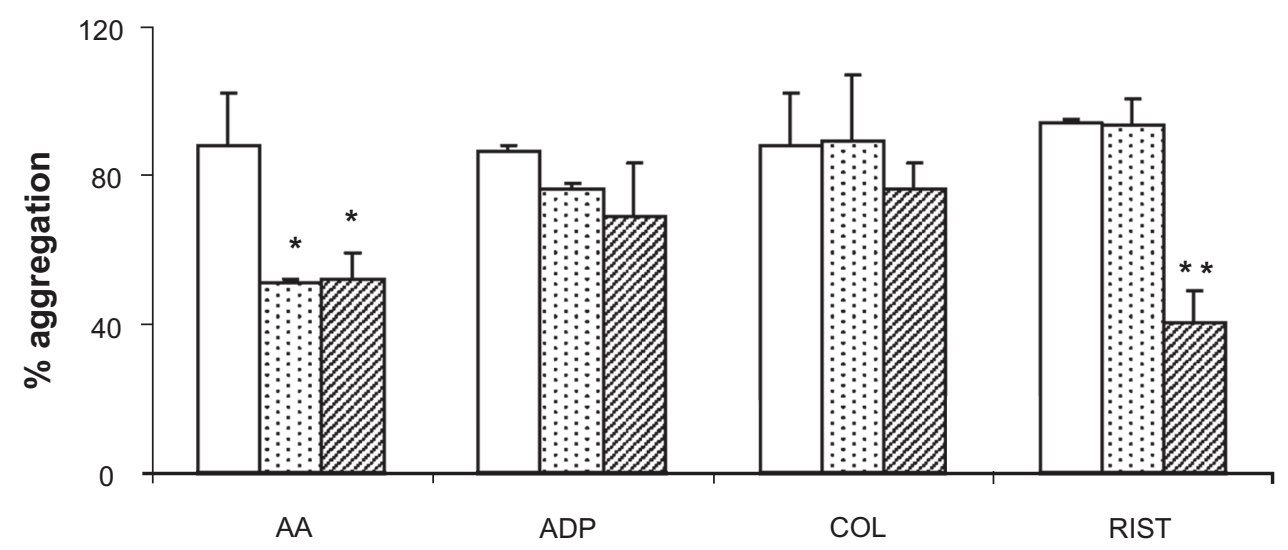

Figure 2 Bar diagram summarizing changes in platelet aggregation induced by different agonists (AA, ADP, COL, and RIST) in samples treated with MLV or MLV-FBN. Results are expressed as mean $\pm S E M ; n=6 ; * P<0.05$; **P $<0.01$.

Abbreviations: AA, arachidonic acid; ADP, adenosine diphosphate; COL, collagen; RIST, ristocetin; MLV, raw multilamellar liposomes; MLV-FBN, fibrinogen-coated liposomes; SEM, standard error of the mean.

In recent years, several groups have explored the use of modified liposomes and microcapsules as coagulants to improve hemostasis. ${ }^{5-9,31,32}$ Our group demonstrated that raw preparations of phospholipids were able to activate the coagulation system and promote fibrin formation on damaged vascular surfaces under thrombopenic conditions. ${ }^{3}$ A reduction of platelet counts is quite common in oncohematological patients who exhibit hemorrhagic episodes that sometimes may require platelet transfusion. Originally, the present study was designed to evaluate the possible effect of FBN-containing liposomes as a prohemostatic strategy in different conditions, such as normal platelet counts and thrombocytopenic conditions, in order to mimic patients potentially requiring platelet transfusion.

Under our experimental conditions, FBN-coated liposomes modified blood coagulation parameters, as indicated by the thromboelastometric and fibrin deposition results. In the FIBTEM thromboelastometric studies, in which platelets were inhibited, MLV-FBN reduced the CT in thrombocytopenic blood, indicating a positive effect on the coagulation mechanism. Interestingly, studies using EXTEM tests on whole blood, when both platelets and the coagulation mechanism are active, have demonstrated a partial inhibition of overall hemostatic parameters that includes a reduction in CFT and clot firmness. Moreover, our results of the experiments under moderate flow conditions indicate that MLV-FBN induced lower clotting activity, as demonstrated by a decrease in fibrin deposition on the subendothelium. Furthermore, MLV-FBN acted on primary hemostasis, as demonstrated by decreased platelet interactions in the perfusion experiments performed at a moderate shear rate. However, no differences were observed at a high shear rate,

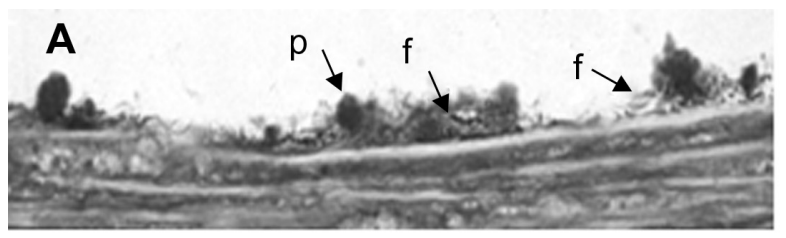

C

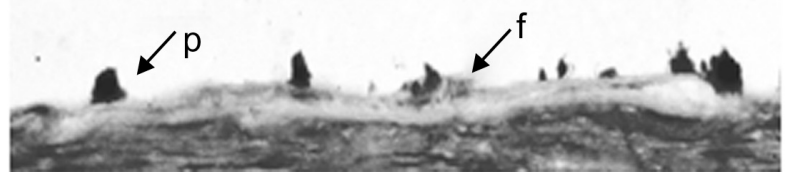

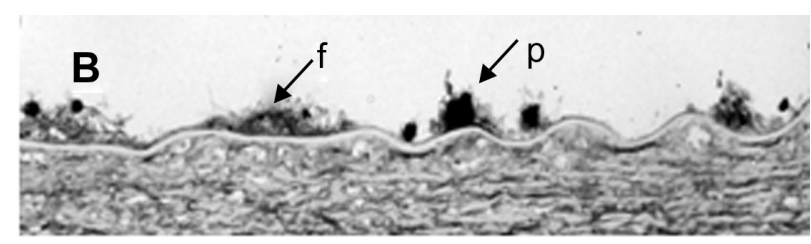

D

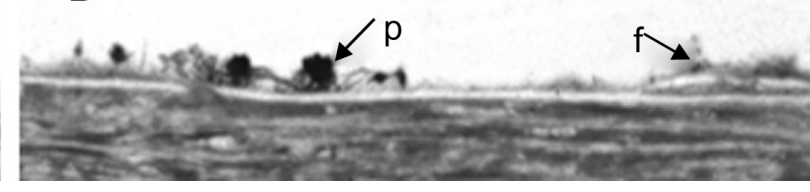

Figure 3 Light micrographs representative of changes induced by MLV-FBN in platelet interaction ( $P$ ) and fibrin deposition (f) on the subendothelium observed in perfusions using whole blood (WB) and thrombocytopenic blood (TPN). (A) WB control; (B) TPN control; (C) WB + MLV-FBN; (D) TPN + MLV-FBN.

Abbreviations: MLV, raw multilamellar liposomes; MLV-FBN, fibrinogen-coated liposomes. 

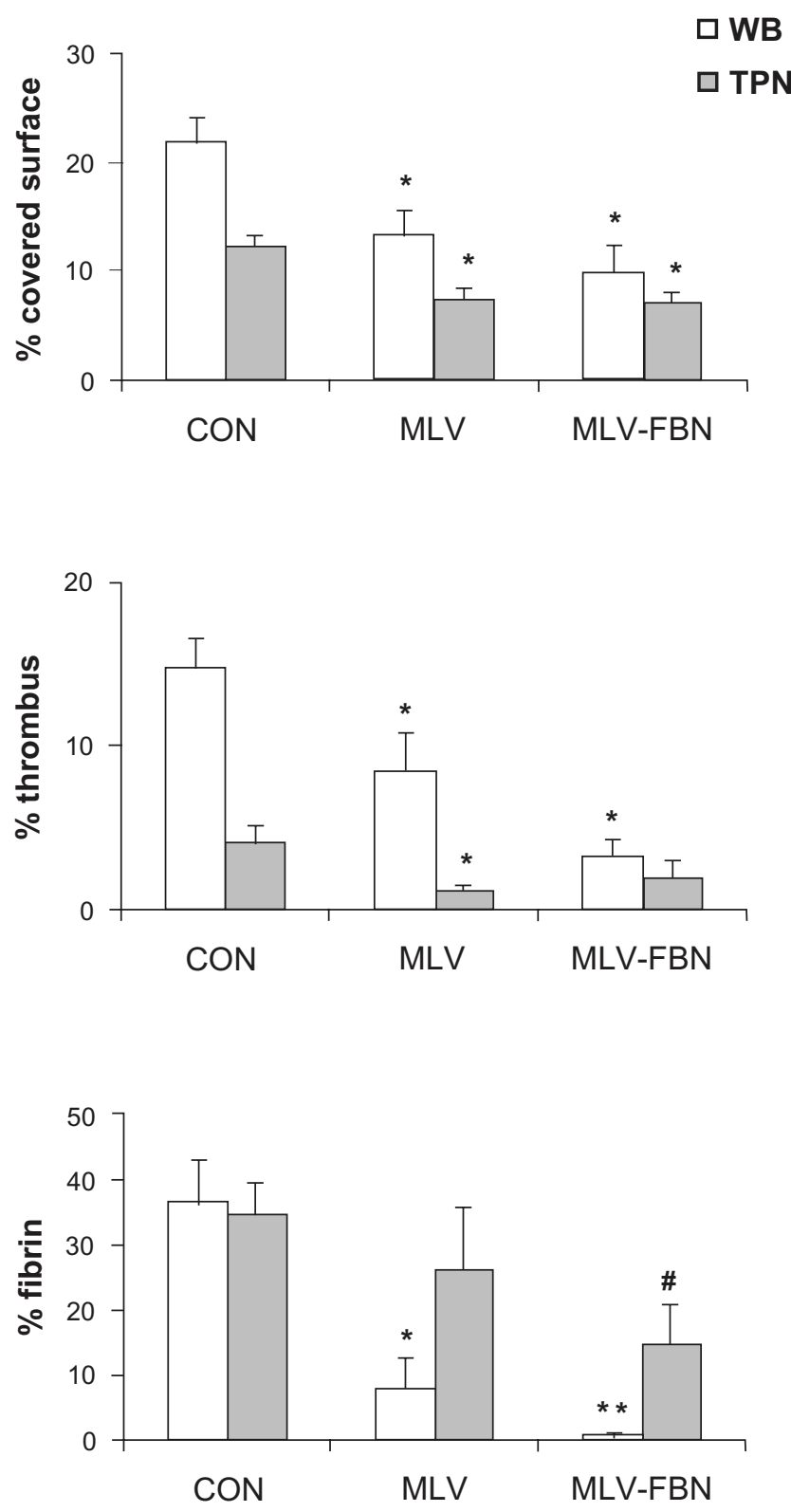

Figure 4 Bar diagram summarizing changes in platelet interaction in samples treated with MLV or MLV-FBN.

Notes: Results are expressed as \% covered surface by platelets, \% of large aggregates (thrombus), and \% of fibrin deposition on the subendothelium. Mean \pm SEM; $n=6$. Comparisons with respect to control: $* \mathrm{P}<0.01$; $* * P<0.005$; comparison of MLV-FBN versus MLV - raw liposomes $\# P<0.01$.

Abbreviations: CON, control; MLV, raw multilamellar liposomes; MLV-FBN, fibrinogen-coated liposomes; SEM, standard error of the mean.

as shown by the closure time obtained with the PFA- $100^{\circledR}$. In fact, it is well established that the shear rate has a role in platelet adhesion and fibrin formation. Under high shear rates, platelet interactions predominate over fibrin formation, whereas under moderate and low shear rates, coagulation mechanisms prevail over platelet interactions. ${ }^{33-35}$ We also observed an inhibition in the aggregation profiles induced by ristocetin and arachidonic acid. In the presence of liposomes, this reduction could be explained by a direct effect of MLV-FBN on the GpIb $\alpha$ complex ${ }^{32}$ in the ristocetin assay and by the phospholipidic composition of the liposomes. Indeed, it has been shown conclusively that the net charge of liposomes and the firmness of the membranes determine the effect on hemostasis. . $^{432,36}$

Although the antihemostatic activity observed in our experiments with FBN-coated liposomes cannot be ascribed to the $\gamma^{\prime}$ chain of FBN, our results (reduced thrombin generation, inhibition of ristocetin-induced aggregation, decrease in fibrin density and inhibition of thrombus formation) show behavior similar to that described for the $\gamma^{\prime}$ chain. ${ }^{11,37}$ This could be explained by a global negative charge in our liposomes ${ }^{17}$ and the use of thiolated FBN during the binding procedure, which could induce residue sulfation and generate conformational changes in the molecule similar to those observed in the $\gamma^{\prime}$ chain. Interestingly, the effect of MLVFBN suggests that with a high number of platelets, FBN acts mainly in primary hemostasis, whereas its participation in coagulation is moderate.

Inconsistent effects of FBN have been described to date, probably because FBN can undergo several posttranslational modifications, such as glycosylation, glycation, nitration, oxidation, sulfation, phosphorylation and proteolytic degradation, and genetic differences among individuals. ${ }^{32}$ In fact, contradictory results in studies on FBN binding to liposomes have been published. Whereas some studies show that conventional approaches to binding proteins to liposomes do not work effectively with $\mathrm{FBN}$ to provide a final product with hemostatic properties ${ }^{38}$ others demonstrate that the binding to liposomes of the dodecapeptide H12, corresponding to a fibrinogen $\gamma$-chain carboxy-terminal sequence, yields a product with demonstrated hemostatic activity. ${ }^{31}$

The biological function of FBN can be modified by changes in protein folding. ${ }^{39}$ It is accepted that structural features of fibrinogen are related to multiple biological functions, including binding to thrombin, fibrinolysis, regulation of FXIII activity, and interactions with blood cells, such as platelets and leukocytes. Residues A414 to L427 of the $\gamma^{\prime}$ chain have been reported to play a central role in thrombin binding because of their overall negative charge, which disrupts the platelet binding domain in the $\gamma \mathrm{A}$ chain and introduces a binding site for zymogen factor XIII and thrombin. ${ }^{10-13}$ Moreover, the sulfation of residues Y418 and Y422 further enhances thrombin binding by increasing the net charge and inhibiting thrombin activity. ${ }^{32,40} \mathrm{On}$ the other hand, $\gamma \mathrm{XL}$ sites in FBN are also involved in the binding to FXIII and to the platelet integrin GPIIb/IIIa. It is not surprizing that when chemical modification occurs, 
the liposomalization of FBN gives liposomes the ability to bind to the GPIIb/IIIa of platelets, thus competitively inhibiting the formation of platelet aggregates. According to Sivaraman and Latour, ${ }^{39}$ the conformation of $\mathrm{FBN}$ is the critical determinant of platelet adhesion. Adsorptioninduced unfolding can expose two distinctly different types of platelet-binding sites in FBN: one induces platelet adhesion alone, and the other induces both platelet adhesion and activation. ${ }^{11,29,41}$

The present study, in contrast to its initial purpose, offers the possibility of developing new clinical strategies for regulating arterial or venous thrombosis based on the use of liposomes. Our data suggest that the antihemostatic effect observed on liposomes is probably related to the conformational structure adopted by the FBN after binding to the liposome. These changes seem to play an important role not only in blood clot formation and stabilization, but also in primary hemostasis. Future investigations will need to address how the binding procedure may interfere with hemostasis.

\section{Acknowledgments}

This work was partially supported by grants PTR95/0890, PET2007_0169, SAF 2009-10365, FIS CP04-00112, FIS PS09/00664, MAT-2009-13155-C04-03, and Red HERACLES RD06/0009 from the Spanish government. Dr Galán belongs to the program of stabilization of researchers in the Instituto de Salud Carlos III, which is funded by the Spanish government, and the Direcció d'Estratègia i Coordinació del Departament de Salut, which is funded by the Generalitat de Catalunya.

\section{Disclosure}

The authors report no conflicts of interest in this work.

\section{References}

1. Streicher P, Nassoy P, Bärmann M, et al. Integrin reconstituted in GUVs: a biomimetic system to study initial steps of cell spreading. Biochim Biophys Acta. 2009;1788:2291-2300.

2. Yatuv R, Robinson M, Dayan-Tarshish I, Baru M. The use of PEGylated liposomes in the development of drug delivery applications for the treatment of hemophilia. Int J Nanomedicine. 2010;5:581-591.

3. Galán AM, Hernández MR, Bozzo J, et al. Preparations of synthetic phospholipids promote procoagulant activity on damaged vessels: studies under flow conditions. Transfusion. 1998;38:1004-1010.

4. Galán AM, Casals E, Estelrich J, et al. Possible hemostatic effect of synthetic liposomes in experimental studies under flow conditions. Haematologica. 2002;87:615-623.

5. Gupta AS, Huang G, Lestini BJ, Sagnella S, Kottke-Marchant K, Marchant RE. RGD-modified liposomes targeted to activated platelets as a potential vascular drug delivery system. Thromb Haemost. 2005;93:106-114.
6. Kitaguchi T, Murata M, Iijima K, Kamide K, Imagawa T, Ikeda Y. Characterization of liposomes carrying von Willebrand factor-binding domain of platelet glycoprotein Ibalpha: a potential substitute for platelet transfusion. Biochem Biophys Res Commun. 1999;261: 784-789.

7. Lee DH, Blajchman MA. Novel treatment modalities: New platelet preparations and substitutes. Br J Haematol. 2001;114:496-505.

8. Levi M, Friederich PW, Middleton S, et al. Fibrinogen-coated albumin microcapsules reduce bleeding in severely thrombocytopenic rabbits. Nat Med. 1999;5:107-111.

9. Rybak ME, Renzulli LA. A liposome based platelet substitute, the plateletsome, with hemostatic efficacy. Biomater Artif Cells Immobilization Biotechnol. 1993;21:101-118.

10. Bertram JP, Williams CA, Robinson R, Segal SS, Flynn NT, Lavik EB. Intravenous hemostat: nanotechnology to halt bleeding. Sci Transl Med. 2009;1:11ra22.

11. Lovely RS, Moaddel M, Farrell DH. Fibrinogen gamma' chain binds thrombin exosite II. J Thromb Haemost. 2003;1:124-131.

12. Takeoka S, Teramura Y, Okamura Y, Handa M, Ikeda Y, Tsuchida E. Fibrinogen-conjugated albumin polymers and their interaction with platelets under flow conditions. Biomacromolecules. 2001;2: 1192-1197.

13. Savage B, Bottini E, Ruggeri ZM. Interaction of integrin alpha IIb beta 3 with multiple fibrinogen domains during platelet adhesion. J Biol Chem. 1995;270:28812-28817.

14. Bennett JS. Platelet-fibrinogen interactions. Ann N Y Acad Sci. 2001;936:340-354.

15. Kirschbaum NE, Mosesson MW, Amrani DL. Characterization of the gamma chain platelet binding site on fibrinogen fragment D. Blood. 1992;79:2643-2648.

16. Hunter RJ. Zeta Potential in Colloid Science. New York: Academic Press, 1981.

17. Casals E, Verdaguer A, Tonda R, Escolar G, Estelrich J. Atomic force microscopy of liposomes bearing fibrinogen. Bioconjugate Chem. 2003;14:593-600.

18. Ellman GL. Tissue sulphydryl groups. Arch Biochem Biophys. 1959;82:70-77.

19. Zwaginga JJ, Sixma JJ, de Groot PG. Activation of endothelial cells induces platelet thrombus formation on their matrix studies of new in vitro thrombosis model with low molecular weight heparin as anticoagulant. Arteriosclerosis. 1990;10:49-61.

20. Alemany M, Hernández MR, Bozzo J, et al. In vitro evaluation of the hemostatic effectiveness of non-viable platelet preparations: studies with frozen-thawed, sonicated or lyophilized platelets. Vox Sang. 1997;73:36-42.

21. Escolar G, Cases A, Viñas M, et al. Evaluation of acquired platelet dysfunctions in uremic and cirrhotic patients using the platelet function analyzer (PFA-100 ${ }^{\mathrm{TM}}$ ): Influence of hematocrit elevation. Haematologica. 1999;84:614-619.

22. Galán AM, Lopez-Vilchez I, Diaz-Ricart M, et al. Serotonergic mechanisms enhance platelet-mediated thrombogenicity. Thromb Haemost. 2009;102:511-519.

23. Sirchia G, Wenz B, Rebulla P, Parravicini A, Carnelli V, Bertolini F. Removal of white cells from red cells by transfusion through a new filter. Transfusion. 1990;30:30-33.

24. Anderson L, Quasim I, Soutar R, Steven M, Macfie A, Korte W. An audit of red cell and blood product use after the institution of thromboelastometry in a cardiac intensive care unit. Transfus Med. 2006;16:31-39.

25. Lak M, Scharling B, Blemings A, et al. Evaluation of rFVIIa (NovoSeven) in Glanzmann patients with thromboelastogram. Haemophilia. 2008;14:103-110.

26. Born JVR, Cross MJ. The aggregation of blood platelets. J Physiol. 1963;168:178-195.

27. Kundu SK, Heilmann EJ, Sio R, Garcia C, Ostgaard RA. Description of an in vitro platelet function analyzer - PFA-100(TM). Seminars in Thrombosis and Hemostasis. 1995;21:106-112. 
28. Baumgartner HR. Platelet interaction with collagen fibrils in flowing blood. Reaction of human platelet with achymotry DPPS in digested subendothelium. Thromb Haemost. 1977;37:1-16.

29. Escolar G, Bastida E, Castillo R, Ordinas A. Development of a computer program to analyze the parameters of platelet-vessel wall interaction. Hemostasis. 1986;16:8-14.

30. Hernández MR, Bozzo J, Mazzara R, Ordinas A, Escolar G. Platelet concentrates promote procoagulant activity: Evidence from experimental studies using a perfusion technique. Transfusion. 1995;35:660-665.

31. Okamura Y, Maekawa I, Teramura Y, et al. Hemostatic effects of phospholipid vesicles carrying fibrinogen gamma chain dodecapeptide in vitro and in vivo. J Thromb Haemost. 2009;7:470-477.

32. Willige SU, Standeven KF, Philippou H, Ariëns RAS. The pleiotropic role of the fibrinogen gamma' chain in hemostasis. Blood. 2009;114:3994-4001.

33. Beumer S, Ijsseldijk MJW, de Groot PG, Sixma JJ. Platelet adhesion to fibronectin in flow: dependence on surface concentration and shear rate, role of platelet membrane glycoproteins GP IIb/IIIa and VLA-5, and inhibition by heparin. Blood. 1994;84:3724-3733.

34. Tonda R, Lopez-Vilchez I, Navalon F, et al. Platelets interact with tissue factor immobilized on surfaces: effects of shear rate. Eur J Clin Invest. 2008;38:34-42.

35. Wu YP, Vink T, Schiphorst M, et al. Platelet thrombus formation on collagen at high shear rates is mediated by von Willebrand factor-glycoprotein Ib interaction and inhibited by von Willebrand factor-glycoprotein IIb/IIIa interaction. Arteriosclerosis Thrombosis and Vascular Biology. 2000;20:1661-1667.
36. An H, Nussio MR, Huson MG, Voelcker NH, Shapter JG. Material properties of lipid microdomains: force-volume imaging study of the effect of cholesterol on lipid microdomain rigidity. Biophysical Journal. 2010;99:834-844.

37. Cooper AV, Standeven KF, Ariëns RA. Fibrinogen gamma-chain splice variant gamma' alters fibrin formation and structure. Blood. 2003;102:535-540.

38. Retzinger GS, Deanglis AP, inventors; University of Cincinnati, assignee. Fibrinogen-coated liposomes. United States patent US 5658588. 1996 Mar 29.

39. Sivaraman B, Latour RA. The relationship between platelet adhesion on surfaces and the structure versus the amount of adsorbed fibrinogen. Biomaterials. 2010;31(5):832-839.

40. Mosesson MW. Update on antithrombin I (fibrin). Thromb Haemost. 2007;98:105-108.

41. Farrell DH, Thiagarajant P, Chung DW, Davie EW. Biochemistry role of fibrinogen a and y chain sites in platelet aggregation. Proc Nati Acad Sci USA. 1992;89:10729-10732.
International Journal of Nanomedicine

\section{Publish your work in this journal}

The International Journal of Nanomedicine is an international, peerreviewed journal focusing on the application of nanotechnology in diagnostics, therapeutics, and drug delivery systems throughou the biomedical field. This journal is indexed on PubMed Central,

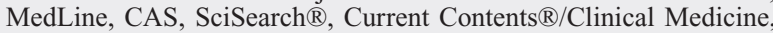

\section{Dovepress}

Journal Citation Reports/Science Edition, EMBase, Scopus and the Elsevier Bibliographic databases. The manuscript management system is completely online and includes a very quick and fair peer-review system, which is all easy to use. Visit http://www.dovepress.com/ testimonials.php to read real quotes from published authors 